\title{
Interference of long non-coding RNA HAGLROS inhibits the proliferation and promotes the apoptosis of ovarian cancer cells by targeting miR-26b-5p
}

\author{
$\mathrm{LIN} \mathrm{ZHU}^{1}$ and MEI MEI ${ }^{2}$ \\ ${ }^{1}$ Department of Gynecology, Beijing Haidian District Maternal and Child Health Hospital, Beijing 100080; \\ ${ }^{2}$ Department of Obstetrics and Gynecology, Xiangyang No. 1 People's Hospital Affiliated to Hubei Medical College, \\ Xiangyang, Hubei 441000, P.R. China
}

Received December 9, 2020; Accepted February 17, 2021

DOI: $10.3892 /$ etm.2021.10311

\begin{abstract}
Ovarian cancer (OV) is the fifth most common type of cancer affecting women worldwide. Long non-coding RNAs (lncRNAs) serve essential roles in the progression of OV. As such, the present study aimed to investigate the specific role of HAGLR opposite strand lncRNA (HAGLROS) in $\mathrm{OV}$ and the underlying mechanism of action through which HAGLROS exerts its effects on OV cells. In the present study, the expression of HAGLROS in several OV cell lines was first detected using reverse transcription-quantitative PCR. HAGLROS was then silenced to evaluate cell viability, proliferation and apoptosis, which were determined using Cell Counting Kit-8, colony formation and TUNEL assays, respectively. Additionally, immunofluorescence staining and western blotting were used to confirm the expression profile of proliferation- and apoptosis-related proteins. Moreover, a dual luciferase reporter assay was used to verify the potential interactions between HAGLROS and microRNA (miR)-26b-5p. Subsequently, rescue assays were performed to investigate the effects of HAGLROS and miR-26b-5p on OV progression. The results indicated that HAGLROS was highly expressed in OV cells. Interference of HAGLROS led to a decrease in the proliferation, but an increase in the apoptosis of OV cells, accompanied by downregulated expression levels of $\mathrm{Ki} 67$ and Bcl-2, and upregulated expression levels of Bax and cleaved caspase-3. Further study revealed that HAGLROS acted as a sponge for miR-26b-5p and positively regulated its expression. miR-26b-5p inhibitor transfection partially reversed the
\end{abstract}

Correspondence to: Dr Mei Mei, Department of Obstetrics and Gynecology, Xiangyang No. 1 People's Hospital Affiliated to Hubei Medical College, 75 Jiefang Road, Xiangyang, Hubei 441000, P.R. China

E-mail: meimeimm2020@126.com

Key words: ovarian cancer, HOXD antisense growth-associated long non-coding RNA opposite strand long non-coding RNA, proliferation, apoptosis, microRNA-26b-5p effects of HAGLROS knockdown on the proliferation and apoptosis of OV cells. In conclusion, the results of the present study suggested that interference of HAGLROS suppressed the proliferation and promoted the apoptosis of OV cells through regulating miR-26b-5p, indicating that HAGLROS may be a promising biomarker in OV diagnosis and treatment.

\section{Introduction}

Ovarian cancer (OV) is the fifth most common type of cancer affecting women worldwide, particularly women aged $>65$ years $(1,2)$. OV is a type of gynecological cancer that is associated with a high morbidity and mortality, severely compromising women's health and quality of life $(3,4)$. Radical surgery and chemotherapy are considered as two important methods for treating OV, but these methods do not appear to reduce the high recurrence rate of $\mathrm{OV}$ or improve the prognosis of the patient with OV (5). The specific pathogenesis behind OV has not been fully elucidated and is incompletely understood. Moreover, patients with OV are commonly diagnosed at an advanced stage, whereby they have missed the opportunity for effective and timely treatment (6). Therefore, the present study aimed to identify potential biomarkers to improve the detection of early stage OV.

Long non-coding RNAs (lncRNAs) are ncRNAs $>200$ nucleotides in length (7-9), which serve important roles in biological processes such as cell differentiation, proliferation, migration and metastasis (10). HOXD antisense growthassociated long non-coding RNA (HAGLR) opposite strand lncRNA (HAGLROS) has been reported to sponge microRNA (miRNA/miR)-15 to accelerate the development of lung cancer and its expression is associated with tumor growth in patients with lung cancer (11). Moreover, HAGLROS has been found to be highly expressed in hepatocellular carcinoma cells, where it promotes the proliferation and reduces the apoptosis of hepatocellular carcinoma cells through regulation of miR-5095/autophagy related 12 signaling (12). Accumulating evidence has indicated that HAGLROS is significantly upregulated in $\mathrm{OV}$ and that it is closely associated with disease stage, tumor size and a poor prognosis for patients with OV (13). 
However, the exact effects of HAGLROS on the progression of OV remain to be elucidated.

miRNAs are RNAs without protein-coding abilities; however, they have been demonstrated to be associated with the proliferation, division, differentiation and apoptosis of various types of cells $(14,15)$. miRNAs are often associated with the development of multiple diseases, including cancer (16). It has been reported that, compared with normal tissues, miR-26b-5p is downregulated in human papillary thyroid cancer tissues (17). miR-26b-5p can inhibit the proliferation, invasion and migration of papillary thyroid cancer cells (17). Emerging evidence has also indicated that miR-26b-5p inhibits the development of multiple myeloma by targeting the Hedgehog signaling pathway (18). Therefore, the present study examined whether HAGLROS exerted any effects on the development of OV by regulating miR-26b-5p.

The present study aimed to evaluate the specific role of HAGLROS in OV and the underlying mechanism of action through which HAGLROS exerts its effects on OV cells. These findings may provide a novel insight into treatment strategies for OV.

\section{Materials and methods}

Cell culture. The normal human ovarian epithelial cell line (IOSE-80) and OV cell lines (ES-2, SKOV3, OVCAR3, HEY and A2780) were purchased from the American Type Culture Collection (ATCC). The information regarding the histotypes of the cell lines is available from the ATCC (https://www. lgcstandards-atcc.org/). The ES-2 cell line was established from a surgical OV tumor specimen. The SKOV3 and OVCAR3 cell lines were derived from a metastatic site: Ascites. The HEY cell line was derived from a peritoneal sample from a patient with moderately differentiated papillary cystadenocarcinoma of the ovary. The A2780 cell line was established from an ovarian endometroid adenocarcinoma tumor from an untreated patient. Cells were cultured in RPMI-1640 medium (Gibco; Thermo Fisher Scientific, Inc.) supplemented with 10\% FBS (Gibco; Thermo Fisher Scientific, Inc.) and incubated at $37^{\circ} \mathrm{C}$ in an incubator containing $5 \% \mathrm{CO}_{2}$.

Cell transfection. SKOV3 cells $\left(1 \times 10^{6}\right.$ cells per well) were plated into 6-well plates and transfection was performed when cells reached the logarithmic growth phase, with $85 \%$ confluence. Two short hairpin (sh)RNAs targeting HAGLROS (shRNA-HAGLROS-1 or shRNA-HAGLROS-2; $1 \mu \mathrm{g}$ ), a scrambled shRNA [shRNA-negative control (NC); $1 \mu \mathrm{g}$ ], miR-26b-5p inhibitor (5'-AAGUUCAUUAAG UCCUAUCCA-3'; $50 \mathrm{nM}$ ) and miR-26b-5p inhibitor NC (inhibitor-NC; 5'-CAGUACUUUUGUGUAGUACAA-3'; $50 \mathrm{nM})$, miR-26b-5p mimic (5'-UUCAAGUAAUUCAGG AUAGGU-3'; $50 \mathrm{nM}$ ) and mimic-NC (5'-UUGUACUAC ACAAAAGUACUG-3'; $50 \mathrm{nM}$ ) were designed and synthesized by Shanghai GenePharma Co., Ltd. Transfection experiments were performed using Lipofectamine ${ }^{\circledR} 2000$ (Invitrogen; Thermo Fisher Scientific, Inc.) at $37^{\circ} \mathrm{C}$ for $48 \mathrm{~h}$, in accordance with the manufacturer's guidelines. Cells were harvested at $48 \mathrm{~h}$ after transfection and the transfection efficiency was determined using reverse transcriptionquantitative PCR (RT-qPCR).
Cell viability assay. A Cell Counting Kit-8 (CCK-8; Shanghai YiSheng Biotechnology Co., Ltd., Shanghai, China) was performed for the detection of cell viability. The transfected SKOV3 cells were plated into $96-$-well plates $(3,000$ cells/well) and incubated for $0,24,48$ and $72 \mathrm{~h}$. Then, CCK-8 solution was added to each well and incubated for another $4 \mathrm{~h}$ at $37^{\circ} \mathrm{C}$. The optical density was measured at $450 \mathrm{~nm}$ using a microplate reader (Bio-Rad Laboratories, Inc.).

Colony formation assay. SKOV3 cells were seeded into 6-well plates (500 cells per well) and incubated for $24 \mathrm{~h}$ to allow for adherence. The medium was changed every 3 days. After 2 weeks, cells were fixed with methanol for $30 \mathrm{~min}$ at room temperature and stained with $0.2 \%$ crystal violet solution for $5 \mathrm{~min}$ at room temperature. Images were captured using a light microscope (magnification, x10; Olympus Corporation).

Immunofluorescence staining. The transfected SKOV3 cells were fixed with $4 \%$ paraformaldehyde at room temperature for $20 \mathrm{~min}$, followed by the addition of $0.1 \%$ Triton X-100 solution at room temperature for $10 \mathrm{~min}$. The cells were blocked with $3 \% \mathrm{BSA}$ solution at $37^{\circ} \mathrm{C}$ for $90 \mathrm{~min}$. Subsequently, a primary antibody against Ki67 (cat. no. 11882S; 1:1,000; Cell Signaling Technology; Boston, MA, USA) was incubated with the cells at $4^{\circ} \mathrm{C}$ overnight, followed by incubation with DyLight ${ }^{\mathrm{TM}}$ 488-conjugated secondary antibody (cat. no. 35553; 1:2,000; Invitrogen; Thermo Fisher Scientific, Inc.) at $37^{\circ} \mathrm{C}$ for $1.5 \mathrm{~h}$ in the dark. Subsequently, DAPI was used to stain the cells for $5 \mathrm{~min}$ at room temperature. After mounting, the cells were observed under a fluorescence microscope (magnification, x200; Olympus Corporation).

TUNEL staining. The transfected SKOV3 cells were fixed with $4 \%$ paraformaldehyde at $4^{\circ} \mathrm{C}$ for $20 \mathrm{~min}$. Cell apoptosis was examined with a TUNEL Apoptosis Detection kit (Invitrogen; Thermo Fisher Scientific, Inc.) at $37^{\circ} \mathrm{C}$ for $1 \mathrm{~h}$, according to the manufacturer's instructions. Subsequently, cells were incubated with diaminobenzene for $10 \mathrm{~min}$ and counterstained with hematoxylin (Beijing Solarbio Science \& Technology Co., Ltd.) for $30 \mathrm{sec}$ at room temperature. The apoptosis of TUNEL-positive cells was observed under an optical microscope (magnification, x200; Olympus Corporation) and cells were counted in five randomly selected microscopic fields.

$R T$ - $q P C R$ assay. Total RNA was extracted from cells utilizing TRIzol $^{\circledR}$ reagent (Invitrogen; Thermo Fisher Scientific, Inc.). cDNA was then synthesized using a SuperScript IV First-Strand Synthesis system (Invitrogen; Thermo Fisher Scientific, Inc.) in accordance with the manufacturer's protocol. Subsequently, using cDNA as the template, the gene expression levels were analyzed using qPCR, which was conducted using iTaq $^{\text {TM }}$ Universal One-Step iTaq ${ }^{\text {TM }}$ Universal SYBR $^{\circledR}$ Green Supermix (Bio-Rad Laboratories, Inc.) on an ABI 7500 instrument (Applied Biosystems; Thermo Fisher Scientific, Inc.). The following thermocycling conditions were used: Initial denaturation at $95^{\circ} \mathrm{C}$ for $10 \mathrm{~min}$; followed by 40 cycles of denaturation at $95^{\circ} \mathrm{C}$ for $15 \mathrm{sec}$ and annealing at $60^{\circ} \mathrm{C}$ for $1 \mathrm{~min}$; and a final extension of $10 \mathrm{~min}$ at $72^{\circ} \mathrm{C}$. Primers used in this study were designed and synthesized by Shenggong Biological Engineering (Shanghai) Co., Ltd. The following 
primers pairs were used: HAGLROS forward, 5'-TCTACA CCCAGAGAGGGACG-3' and reverse, 5'-GCCTACTTCCTC CCACACAA-3'; GAPDH forward, 5'-ACAACTTTGGTA TCGTGGAAGG-3' and reverse, 5'-GCCATCACGCCACAG TTTC-3'; miR-26b-5p forward, 5'-TTCAAGTAATTCAGG ATAGGT-3' and reverse, 5'-GTGCGTGTCGTGGAGTC-3'; U6 forward, 5'-GGAACGATACAGAGAAGATTAGC-3' and reverse, 5'-TGGAACGCTTCACGAATTTGCG-3'. GAPDH or U6 were used as internal controls for normalization. The $2^{-\Delta \Delta C q}$ method was used to calculate relative RNA expression (19).

Western blot analysis. Cellular proteins were isolated from cells using RIPA buffer (Beyotime Institute of Biotechnology). The protein quantification was conducted using a BCA kit. Subsequently, equal amounts of proteins (40 $\mu \mathrm{g}$ /lane) were separated on $10 \%$ SDS-PAGE gels and transferred to PVDF membranes (EMD Millipore), which were then blocked with $5 \%$ skimmed milk for $2 \mathrm{~h}$ at $37^{\circ} \mathrm{C}$. Primary antibodies against Bcl-2 (1:1,000; cat. no. 15017; Cell Signaling Technology, Inc.), Bax (1:1,000; cat. no. 14796; Cell Signaling Technology, Inc.), cleaved-caspase 3 (1:1,000; cat. no. 9664; Cell Signaling Technology, Inc.) and GAPDH (1:1,000; cat. no. 5174; Cell Signaling Technology, Inc.) were incubated with the membranes at room temperature overnight. Subsequently, the membranes were incubated with the horseradish peroxidase-conjugated goat anti-rabbit secondary antibody (1:5,000; cat. no. abs20002; Absin Bioscience, Inc.) at $37^{\circ} \mathrm{C}$ for $1 \mathrm{~h}$. The bands were detected using an ECL reagent (EMD Millipore). The grayscale values of the membranes were semi-quantified using ImageJ software (version 1.52r; National Institutes of Health). GAPDH was used as an internal control.

Luciferase reporter assay. The target miRNA of HAGLROS was predicted using the DIANA database (http://carolina.imis.athena-innovation.gr/diana_tools/web/index. php? $r=$ lncbasev2\%2Findex), a database that predicts the binding of lncRNAs and miRNAs (20). A dual luciferase reporter assay system (Promega Corporation) was used to verify the interaction between the HAGLROS and miR-26b-5p. The full-length of the wild-type (WT) HAGLROS 3'-untranslated region (3'-UTR) (HAGLROS-WT) (Shanghai GenePharma Co., Ltd.) with the putative binding sites of miR-26b-5p was inserted into firefly luciferase reporter and then transfected into SKOV3 cells using Lipofectamine ${ }^{\circledR} 2000$ (Invitrogen; Thermo Fisher Scientific, Inc.). Constructs containing the mutated putative binding sequence of miR-26b-5p located within the 3'-UTR of HAGLROS (HAGLROS-MUT) were considered as mutated control and mutated controls were also cloned into SKOV3 cells. After incubation for $48 \mathrm{~h}$ at $37^{\circ} \mathrm{C}$, firefly and Renilla luciferase activities were assessed.

Statistical analysis. GraphPad Prism version 8.0 (GraphPad Software, Inc.) was used to analyze the experimental data. All experiments were performed three times and the data were presented as mean \pm SD. Comparisons between two groups were conducted using unpaired student's t-tests and comparisons between multiple groups were performed using one-way ANOVAs with Tukey's post hoc tests. $\mathrm{P}<0.05$ was considered to show statistically significant.

\section{Results}

Interference of IncRNA HAGLROS inhibits the proliferation of $O \mathrm{~V}$ cells. The expression levels of HAGLROS were detected using RT-qPCR in normal human ovarian epithelial cells (IOSE-80) and OV cell lines (ES-2, SKOV3, OVCAR3, HEY and A2780). As presented in Fig. 1A, the expression levels of HAGLROS were notably upregulated in OV cells, compared with IOSE- 80 cells. The highest expression levels of HAGLROS were found in SKOV3 cells, thus SKOV3 cells were selected for further experiments. The aforementioned results also indicated that HAGLROS may act as a tumor suppressor in OV. Therefore, HAGLROS was knocked down through transfections with shRNA-HAGLROS-1 and shRNA-HAGLROS-2. HAGLROS expression was significantly decreased following transfection and shRNA-HAGLROS-2, which displayed a lower expression of HAGLROS, was selected for the following experiments (Fig. 1B). Subsequently, it was observed that the cell viability and colony formation abilities were markedly suppressed by the knockdown of HAGLROS compared with the shRNA-NC group in SKOV3 cells (Fig. 1C and D). Consistently, the expression levels of $\mathrm{Ki} 67$, a proliferation-related protein, was decreased in the HAGLROS-knockdown group, compared with those in the shRNA-NC group (Fig. 1E). Overall, these data provided evidence that knockdown of HAGLROS inhibited the proliferation of OV cells.

Interference of HAGLROS promotes the apoptosis of OV cells. The apoptosis of OV cells was detected using a TUNEL assay to confirm whether knockdown of HAGLROS could affect the apoptosis of OV cells. The TUNEL assay results demonstrated that the apoptosis of OV cells in the shRNA-HAGLROS group was notably increased compared with OV cells in the shRNA-NC group (Fig. 2A and B). Furthermore, the expression levels of the anti-apoptotic protein Bcl-2 were downregulated, while those of the pro-apoptotic proteins Bax and cleaved-caspase 3 were upregulated after HAGLROS knockdown, compared with the shRNA-NC group (Fig. 2C). These results suggested that interference of HAGLROS promoted the apoptosis of OV cells.

HAGLROS is directly targeted by miR-26b-5p. The DIANA database (http://carolina.imis.athenainnovation.gr/diana_ tools/web/index.php? $\mathrm{r}=$ lncbasev2\%2Findex) predicted that HAGLROS could target miR-26b-5p. The expression of miR-26b-5p was determined using RT-qPCR in a normal human ovarian epithelial cell line and OV cell lines. It was found that miR-26b-5p was downregulated in $\mathrm{OV}$ cell lines compared with normal human ovarian epithelial cells (Fig. 3A). The binding sites between HAGLROS and miR-26b-5p are presented in Fig. 3B. Significantly upregulated miR-26b-5p expression levels were observed after transfection with miR-26b-5p mimic as compared to the mimic-NC group (Fig. 3C). Dual luciferase reporter assay results suggested that the luciferase activity in the miR-26b-5p mimic + HAGLROS-WT group was weaker compared with other groups, indicating the regulatory relationship between HAGLROS and miR-26b-5p (Fig. 3D). Additionally, HAGLROS-knockdown markedly increased the expression levels of miR-26b-5p in SKOV3 cells as compared with the 

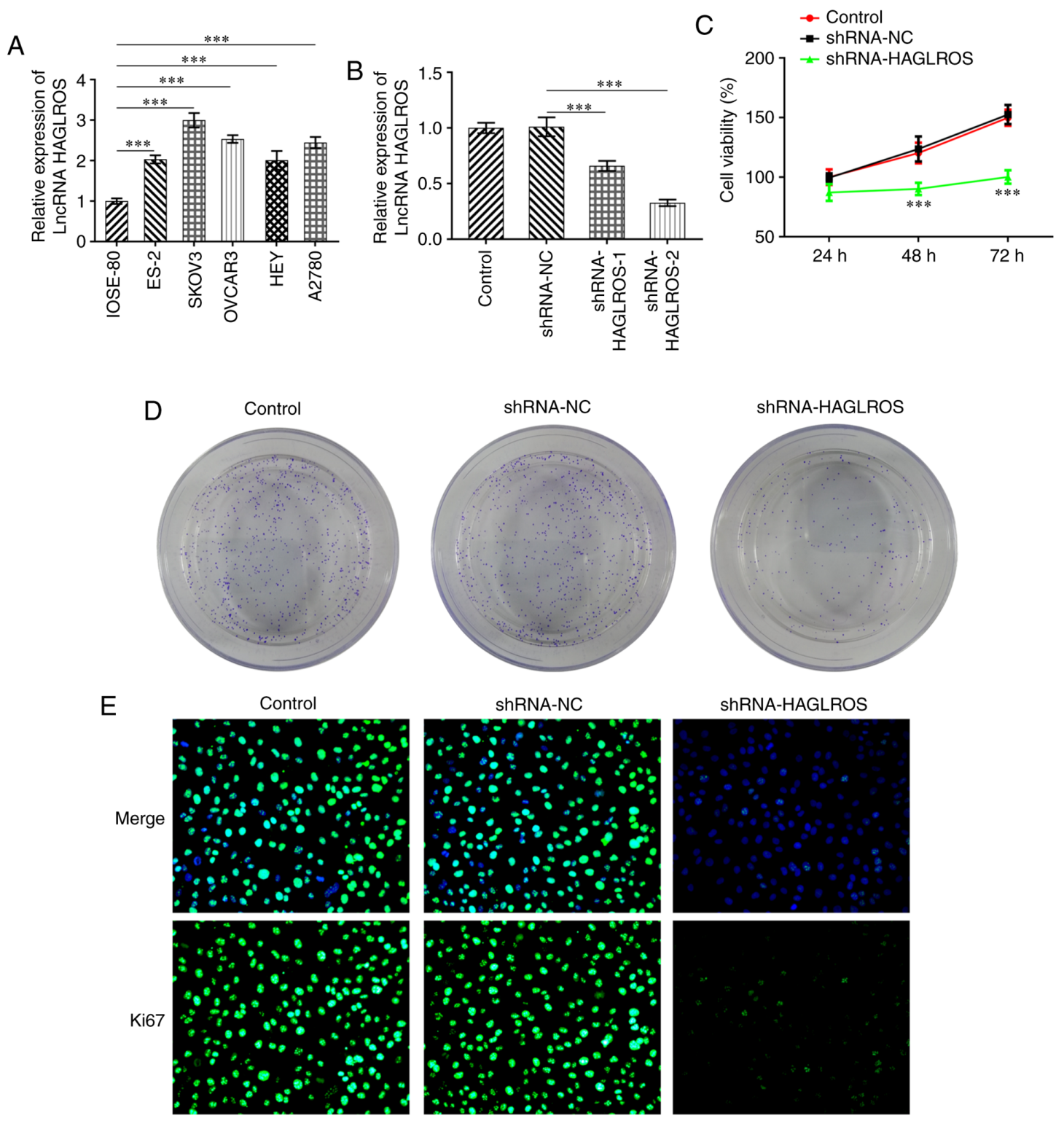

ShRNA-HAGLROS
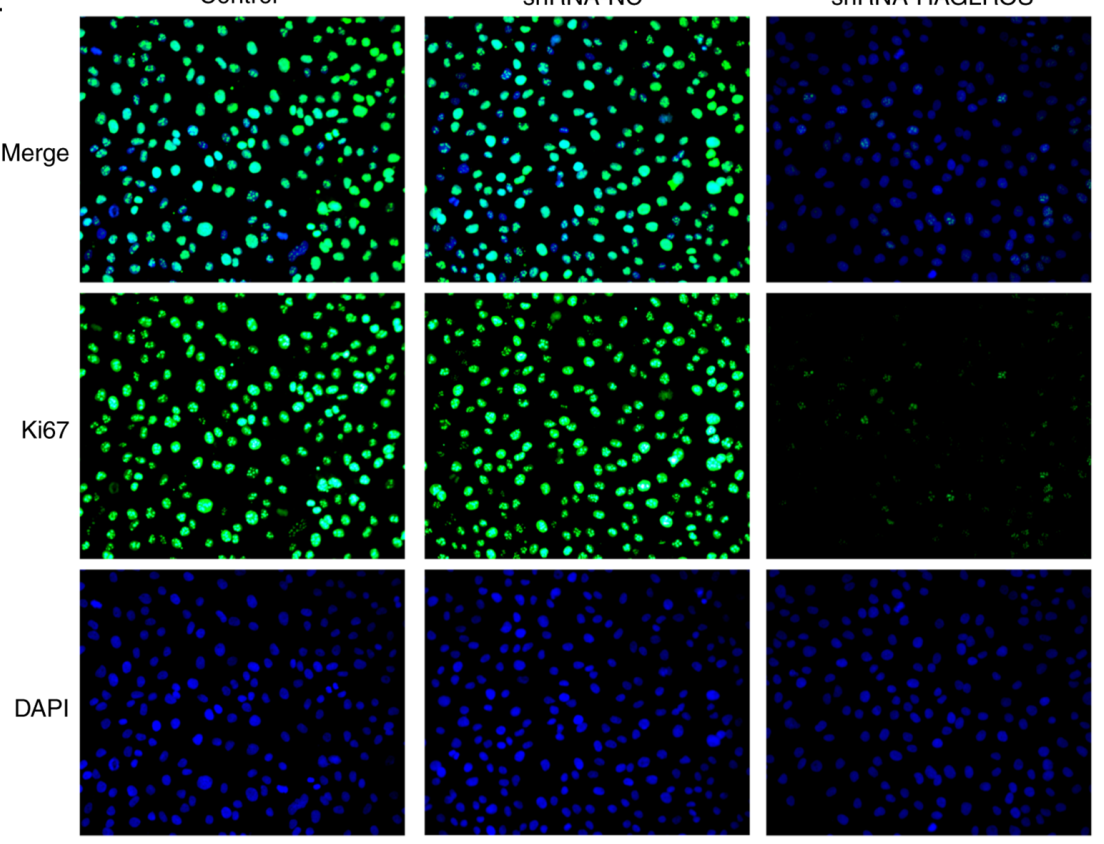

Figure 1. Interference of HAGLROS inhibits the proliferation of OV cells. (A) RT-qPCR was used to detect the expression levels of HAGLROS in several OV cell lines (ES-2, SKOV3, OVCAR3, HEY and A2780) and the normal human ovarian epithelial cell line IOSE-80. ${ }^{* * *}$ P $<0.001$. (B) RT-qPCR was used to evaluate the transfection efficiency of HAGLROS knockdown plasmids. ${ }^{* * *} \mathrm{P}<0.001$. (C) Cell viability was examined using a Cell Counting Kit- 8 assay. ${ }^{* * * *} \mathrm{P}<0.001$ vs. shRNA-NC. (D) Cell proliferation was assessed using a colony formation assay. (E) Ki67 expression was determined using immunofluorescence staining (magnification, x200). HAGLROS, HOXD antisense growth-associated long non-coding RNA opposite strand lncRNA; IncRNA, long non-coding RNA; NC, negative control; OV, ovarian cancer; RT-qPCR, reverse transcription-quantitative PCR; shRNA, short hairpin RNA.

shRNA-NC group (Fig. 3E). Thus, these results suggested that HAGLROS may bind directly to miR-26b-5p.

Interference of HAGLROS suppresses the proliferation and promotes the apoptosis of OV cells by regulating miR-26b-5p. As the regulatory relationship between HAGLROS and miR-26b-5p was identified, it was subsequently investigated whether interference of HAGLROS could inhibit the proliferation and promote the apoptosis of OV cells by targeting
miR-26b-5p. First, the miR-26b-5p inhibitor was used to decrease the expression levels of miR-26b-5p (Fig. 4A). The proliferative (Fig. 4B) and colony formation (Fig. 4C) abilities of SKOV3 cells transfected with shRNA-HAGLROS were found to be markedly decreased, which was reversed by the miR-26b-5p inhibitor.Consistently, the expression of Ki67 was notably downregulated in the shRNA-HAGLROS compared with the shRNA-NC group, whereas miR-26b-5p-knockdown attenuated this effect (Fig. 4D). Additionally, the TUNEL 

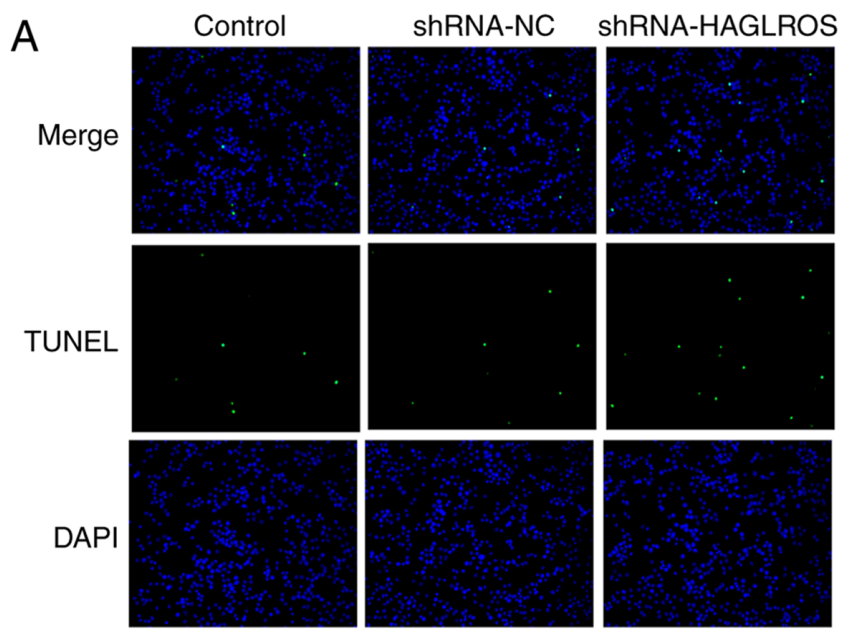

B
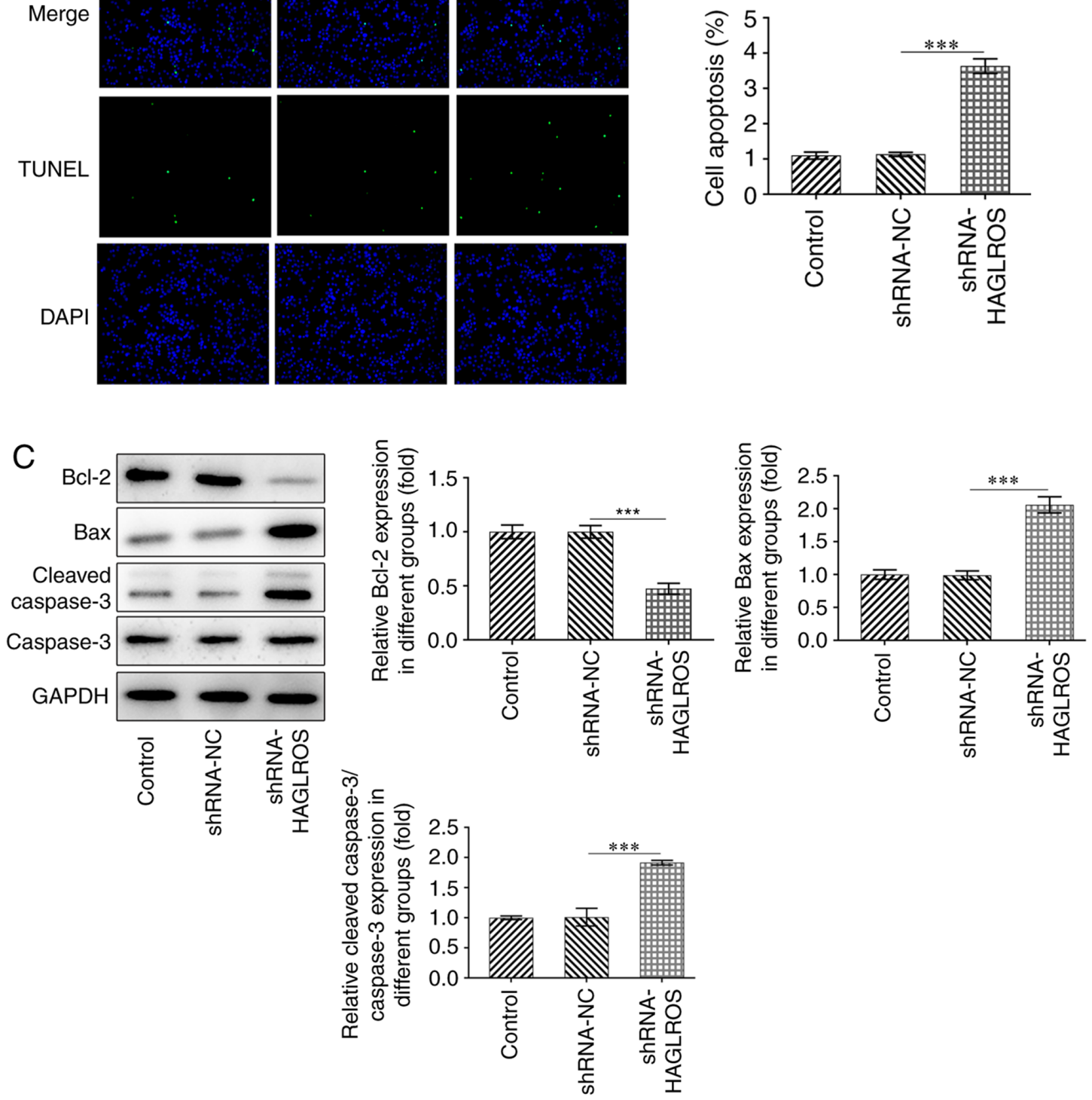

Figure 2. HAGLROS silencing promotes the apoptosis of ovarian cancer cells. (A and B) Apoptosis of SKOV3 cells was detected using TUNEL staining after knocking down HAGLROS (magnification, x200). (C) Western blot analysis was used to detect the expression levels of apoptosis-related proteins in SKOV3 cells after HAGLROS silencing. ${ }^{* * *} \mathrm{P}<0.001$. HAGLROS, HOXD antisense growth-associated long non-coding RNA opposite strand long non-coding RNA; shRNA, short hairpin RNA.

staining (Fig. 4E and F) results indicated that the impact of HAGLROS knockdown on the apoptosis of SKOV3 cells was partially counteracted by the miR-26b-5p inhibitor. Meanwhile, co-transfection with shRNA-HAGLROS and miR-26b-5p inhibitor led to a significant upregulation of Bcl-2 protein expression levels, as well as a downregulation of Bax and cleaved caspase- 3 protein expression levels (Fig. 4G). Collectively, these findings demonstrate that interference of HAGLROS inhibits the proliferation and promotes the apoptosis of SKOV3 cells by regulating miR-26b-5p.

\section{Discussion}

Accumulating evidence has indicated that lncRNAs are involved in tumorigenesis and have the potential to be used as diagnostic and prognostic biomarkers in a wide range of cancer types $(21,22)$. The major finding of the present study was that HAGLROS was notably upregulated in OV tissues and cells, and that HAGLROS-knockdown inhibited the proliferation and promoted the apoptosis of OV cells by sponging miR-26b-5p.

lncRNAs, which can serve as critical tumor promoters or suppressors, can affect the occurrence and progression of multiple types of diseases $(23,24)$. HAGLROS is abnormally expressed in the serum of patients with pneumonia and it has been found to decrease apoptosis and autophagy in lipopolysaccharide-induced WI-38 cells (25). Previous studies have also reported the importance of HAGLROS in the development of various types of cancer $(11,26)$. For instance, HAGLROS, which was found to be upregulated in gastric cancer, is an indicator for the overall survival of patients with gastric cancer (27). High expression levels of HAGLROS has 


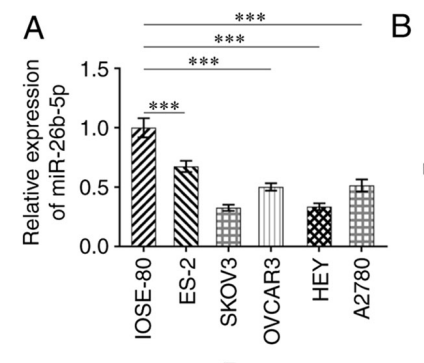

$\mathrm{D}$

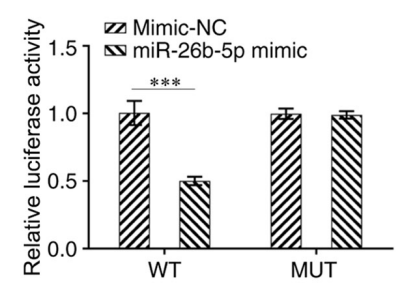

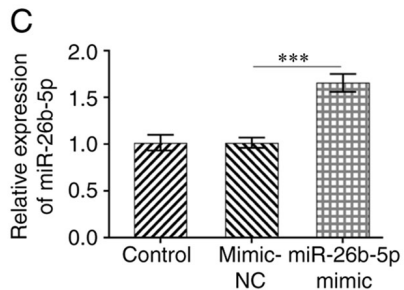

E

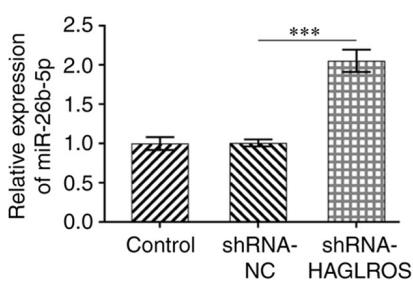

Figure 3. HAGLROS is directly targeted by miR-26b-5p. (A) RT-qPCR was used to determine the expression levels of miR-26b-5p in normal human ovarian epithelial cells and several OV cell lines. (B) Potential binding sites between HAGLROS and miR-26b-5p were predicted. (C) The expression levels of miR-26b-5p was tested using RT-qPCR. (D) Dual luciferase reporter assays were used to verify the binding association between HAGLROS and miR-26b-5p. (E) miR-26b-5p expression was detected after interference of HAGLROS in OV cells. ${ }^{* * *} \mathrm{P}<0.001$. HAGLROS, HOXD antisense growth-associated long non-coding RNA opposite strand long non-coding RNA; miR, microRNA; MUT, mutant; NC, negative control; OV, ovarian cancer; RT-qPCR, reverse transcription quantitative PCR; shRNA, short hairpin RNA; WT, wild-type.

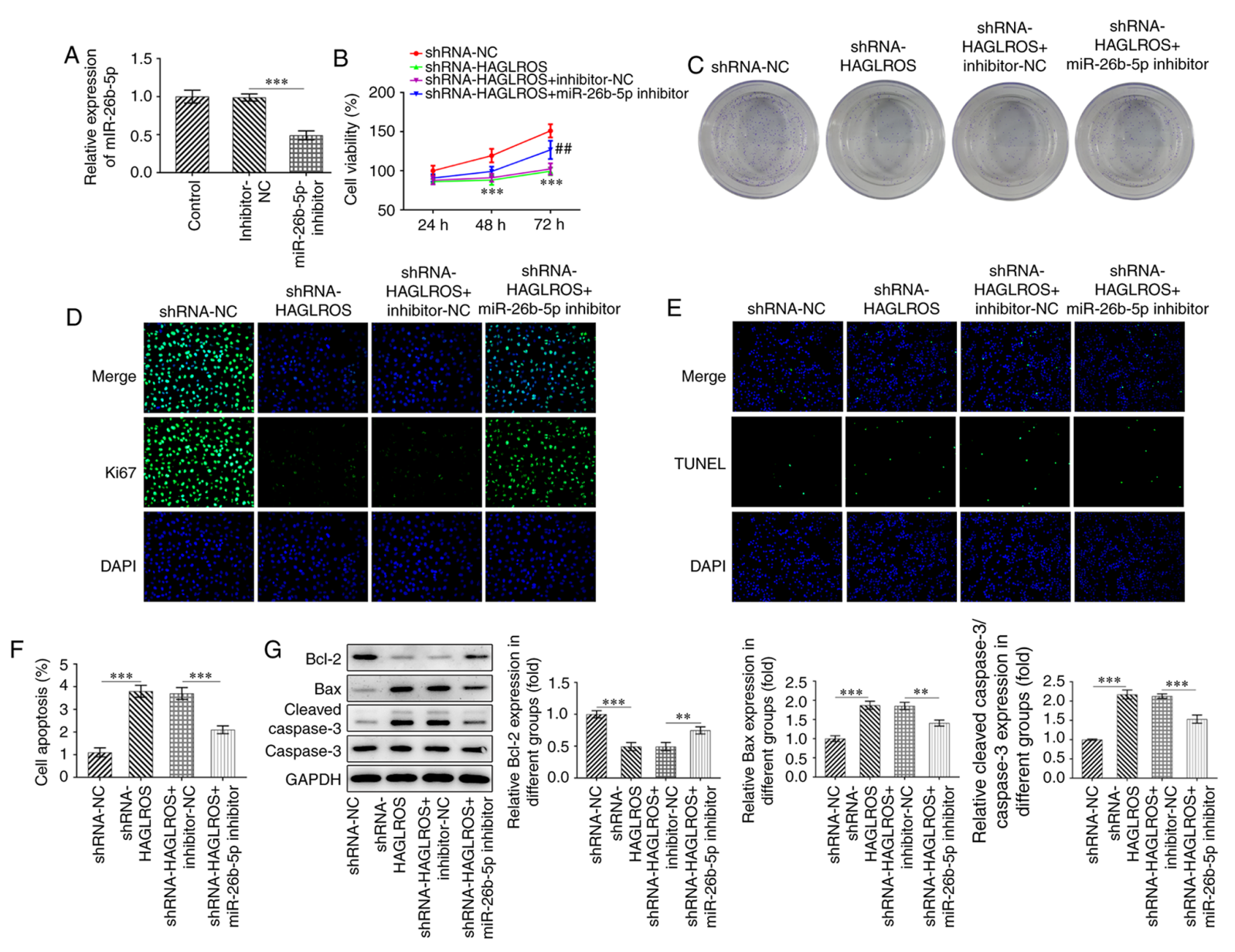

Figure 4. HAGLROS silencing inhibits the proliferation and promotes the apoptosis of OV cells by regulating miR-26b-5p. (A) Reverse transcriptionquantitative PCR was used to detect the expression levels of miR-26b-5p after transfection with miR-26b-5p inhibitor. ${ }^{* * *} \mathrm{P}<0.001$. OV cell (B) viability and

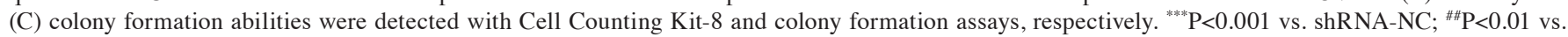
shRNA-HAGLROS + inhibitor-NC. (D) Ki67 expression was evaluated with immunofluorescence staining (magnification, x200). (E and F) Apoptosis of SKOV3 cells was determined using TUNEL staining. (G) Western blot analysis was used to detect the expression levels of apoptosis-related proteins in OV cells. ${ }^{* *} \mathrm{P}<0.01,{ }^{* * * *} \mathrm{P}<0.001$. HAGLROS, HOXD antisense growth-associated long non-coding RNA opposite strand long non-coding RNA; miR, microRNA; $\mathrm{NC}$, negative control; OV, ovarian cancer; shRNA, short hairpin RNA. 
also been closely associated with the short overall survival time of patients with non-small cell lung cancer (28). Moreover, HAGLROS has been found to be elevated in patients with $\mathrm{OV}$ at advanced stages and its expression is considered to be associated with a poor prognosis for patients with OV (13). Therefore, HAGLROS is of great clinical significance for the diagnosis and treatment of OV. In the present study, HAGLROS was highly expressed in OV cells, which was in line with the findings of the previous study (13). Furthermore, OV cells had presented with reduced proliferation and enhanced apoptosis after knockdown of HAGLROS.

Mechanically, it is known that lncRNAs function as competing endogenous RNAs to regulate tumor progression by sponging specific miRNAs $(29,30)$. miRNAs are important in gene regulation and cellular processes in various types of diseases (31). In the present study, a binding site for miR-26b-5p was identified in the sequence of HAGLROS and the dual-luciferase reporter assay confirmed that HAGLROS could directly target miR-26b-5p.

miR-26 consists of four highly conserved small non-coding RNAs that share similarity in structures and sequences (32). miR-26b, a member of the miR-26 family, has been considered as an effective marker in the diagnosis and treatment of breast cancer, gastric cancer and OV $(33,34)$. With regards to the functions of miR-26b-5p in cancer, accumulating evidence has suggested that miR-26b-5p may inhibit cancer progression in prostate cancer, thyroid cancer and head and neck squamous cell carcinoma $(35,36)$. Moreover, a previous study revealed that the expression of miR-26b-5p was reduced in bladder cancer tissues and cells compared with that in adjacent bladder tissues, and that miR-26b-5p acted as a tumor suppressor in bladder cancer (31). Additionally, miR-26b-5p has been reported to play a protective effect on cis-diamine dichloroplatinum-induced ovarian granulosa cells through targeting mitogen-activated protein kinase kinase kinase 9 (37). In the present study, a series of functional experiments indicated a regulatory association between HAGLROS and miR-26b-5p. The results suggested that HAGLROS was inversely associated with the expression of miR-26b-5p. It was also identified that the miR-26b-5p inhibitor abolished the anti-proliferative and pro-apoptotic effects of HAGLROS in OV cells.

In conclusion, to the best of our knowledge, the present results provided the first evidence that HAGLROS is notably upregulated in OV cells and that interference of HAGLROS inhibited the proliferation and promoted the apoptosis of $\mathrm{OV}$ cells through regulating miR-26b-5p. As such, these findings may provide researchers with valuable additional insights into an in-depth understanding of the underlying mechanisms of action for the HAGLROS/miR-26b-5p axis in OV and indicate the potential value of HAGLROS as a promising biomarker for the diagnosis and treatment of OV. The specific signaling pathway or specific signaling proteins regulated by HAGLROS and miR-26b-5p, the expression levels of HAGLROS in the different histotypes of OV and different OV cell lines should be investigated in further experiments, the lack of which are limitations of the present study.

\section{Acknowledgements}

Not applicable.

\section{Funding}

No funding was received.

\section{Availability of data and materials}

The datasets used and/or analyzed during the current study are available from the corresponding author on reasonable request.

\section{Authors' contributions}

LZ and MM searched the literature, designed the experiments and performed the experiments. LZ analyzed, interpreted the data and wrote the manuscript. MM revised the manuscript. LZ and MM confirmed the authenticity of all the raw data. All authors read and approved the final manuscript.

\section{Ethics approval and consent to participate}

Not applicable.

\section{Patient consent for publication}

Not applicable.

\section{Competing interests}

The authors declare that they have no competing interests.

\section{References}

1. Sullivan R: Cancer research in the UK: A policy review of the junior academic clinical faculty. Mol Oncol 1: 366-373, 2008

2. Rooth C: Ovarian cancer: Risk factors, treatment and management. Br J Nurs 22 (Suppl 17): S23-S30, 2013.

3. Lin X, Feng D, Li P and Lv Y: LncRNA LINC00857 regulates the progression and glycolysis in ovarian cancer by modulating the Hippo signaling pathway. Cancer Med 9: 8122-8132, 2020.

4. Torre LA, Bray F, Siegel RL, Ferlay J,Lortet-Tieulent J and Jemal A: Global cancer statistics, 2012. CA Cancer J Clin 65: 87-108, 2015.

5. Jelovac D and Armstrong DK: Recent progress in the diagnosis and treatment of ovarian cancer. CA Cancer J Clin 61: 183-203, 2011.

6. Lou Y, Jiang H, Cui Z, Wang X, Wang L and Han Y: Gene microarray analysis of lncRNA and mRNA expression profiles in patients with highgrade ovarian serous cancer. Int J Mol Med 42: 91-104, 2018.

7. Zhang M, Song Y and Yu L: LncRNA PTCSC3 suppressed cervical carcinoma cell invasion and proliferation via regulating miR-574-5p. Am J Transl Res 11: 7186-7194, 2019.

8. Yuan L, Ma T, Liu W, Chen Y, Yuan Q, Ye M, Yu L, Li J, Niu Y and Nan Y: LINC00994 promoted invasion and proliferation of gastric cancer cell via regulating miR-765-3p. Am J Transl Res 11: 6641-6649, 2019.

9. Yin X, Zhang J, Li C, Zhang Z, Jin T, Song L, Zhang R, Wang W, Tao Y and Wang X: LncRNA HOXA11-AS accumulation-induced microRNA-761 downregulation regulates cell growth by targeting TRIM29 in papillary thyroid cancer. Am J Transl Res 11: 6826-6837, 2019.

10. Lei X, Yang S, Yang Y, Zhang J, Wang Y and Cao M: Long noncoding RNA DLX6-AS1 targets miR-124-3p/CDK4 to accelerate Ewing's sarcoma. Am J Transl Res 11: 6569-6576, 2019.

11. Wang WL, Yu DJ and Zhong M: LncRNA HAGLROS accelerates the progression of lung carcinoma via sponging microRNA-152. Eur Rev Med Pharmacol Sci 23: 6531-6538, 2019.

12. Wei H, Hu J, Pu J, Tang Q, Li W, Ma R, Xu Z, Tan C, Yao T, Wu X, et al: Long noncoding RNA HAGLROS promotes cell proliferation, inhibits apoptosis and enhances autophagy via regulating miR-5095/ATG12 axis in hepatocellular carcinoma cells. Int Immunopharmacol 73: 72-80, 2019. 
13. Yang M,Zhai Z,Zhang Y and Wang Y: Clinical significance and oncogene function of long noncoding RNA HAGLROS overexpression in ovarian cancer. Arch Gynecol Obstet 300: 703-710, 2019.

14. Han G, Qiu N, Luo K, Liang H and Li H: Downregulation of miroRNA-141 mediates acquired resistance to trastuzumab and is associated with poor outcome in breast cancer by upregulating the expression of ERBB4. J Cell Biochem: Feb 11, 2019 (Epub ahead of print).

15. Yu FQ, Wang Z, Wang XW, Wang SL, Li XD, Huang QS and Lin JH: MicroRNA-885-5p promotes osteosarcoma proliferation and migration by downregulation of cell division cycle protein 73 homolog expression. Oncol Lett 17: 1565-1572, 2019.

16. Dejene SB, Ohman AW, Du W, Randhawa D, Bradley A, Yadav N, Elias KM, Dinulescu DM and Setlur SR: Defining fallopian tube-derived miRNA cancer signatures. Cancer Med 8: 6709-6716, 2019.

17. Zhou A, Pan H, Sun D, Xu H, Zhang C, Chen X, Li L and Wang T: miR-26b-5p inhibits the proliferation, migration and invasion of human papillary thyroid cancer in a $\beta$-catenin-dependent manner. Onco Targets Ther 13: 1593-1603, 2020.

18. Jia CM, Tian YY, Quan LN, Jiang L and Liu AC: miR-26b-5p suppresses proliferation and promotes apoptosis in multiple myeloma cells by targeting JAG1. Pathol Res Pract 214: 1388-1394, 2018.

19. Livak KJ and Schmittgen TD: Analysis of relative gene expression data using real-time quantitative PCR and the 2(-Delta Delta C(T)) method. Methods 25: 402-408, 2001.

20. Paraskevopoulou MD, Vlachos IS, Karagkouni D, Georgakilas G Kanellos I, Vergoulis T, Zagganas K, Tsanakas P, Floros E, Dalamagas T and Hatzigeorgiou AG: DIANA-LncBase v2: Indexing microRNA targets on non-coding transcripts. Nucleic Acids Res 44: D231-D238, 2016.

21. Xu HY, Wang LY and Jiang XL: Silencing of 1ncRNA DLEU1 inhibits tumorigenesis of ovarian cancer via regulating miR-429/TFAP2A axis. Mol Cell Biochem 476: 1051-1061, 2021.

22. Du LJ, Mao LJ and Jing RJ: Long noncoding RNA DNAH17-AS1 promotes tumorigenesis and metastasis of non-small cell lung cancer via regulating miR-877-5p/CCNA2 pathway. Biochem Biophys Res Commun 533: 565-572, 2020.

23. Zhang XJ, Qi GT, Zhang XM, Wang L and Li FF: lncRNA RHPN1-AS1 promotes the progression of endometrial cancer through the activation of ERK/MAPK pathway. J Obstet Gynaecol Res 47: 533-543, 2021.

24. Yao HP, Chen R, Yang YX and Jiang J: LncRNA BBOX1-AS1 aggravates the development of ovarian cancer by sequestering miR-361-3p to augment PODXL expression. Reprod Sci 28 736-744, 2021.

25. Liu MH, Han T, Shi SM and Chen EQ: Long noncoding RNA HAGLROS regulates cell apoptosis and autophagy in lipopolysaccharides-induced WI-38 cells via modulating miR-100/ NF-kappa B axis. Biochem Biophys Res Commun 500: 589-596, 2018.
26. Zhou KF, Xu J, Yin XF and Xia JN: Long noncoding RNA HAGLROS promotes cell invasion and metastasis by sponging miR-152 and upregulating ROCK1 expression in osteosarcoma. Comput Math Method Med 2020: 7236245, 2020.

27. Chen JF, Wu P, Xia R, Yang J, Huo XY, Gu DY, Tang CJ, De W and Yang F: STAT3-induced lncRNA HAGLROS overexpression contributes to the malignant progression of gastric cancer cells via mTOR signal-mediated inhibition of autophagy. Mol Cancer 17: 6, 2018.

28. Chen Y, Shen T, Ding X, Cheng L, Sheng L and Du X: HAGLROS is overexpressed and promotes non-small cell lung cancer migration and invasion. Jpn J Clin Oncol 50: 1058-1067, 2020.

29. Liu XH, Sun M, Nie FQ, Ge YB, Zhang EB, Yin DD, Kong R, Xia R, Lu KH, Li JH, et al: Lnc RNA HOTAIR functions as a competing endogenous RNA to regulate HER 2 expression by sponging miR-331-3p in gastric cancer. Mol Cancer 13: 92, 2014

30. Chen $P$, Zhao X, Wang H, Zheng M, Wang Q and Chang W: The down-regulation of lncRNA PCAT18 promotes the progression of gastric cancer via MiR-107/PTEN/PI3K/AKT signaling pathway. Onco Targets Ther 12: 11017-11031, 2019.

31. Wu K, Mu XY, Jiang JT, Tan MY, Wang RJ, Zhou WJ, Wang X, He YY, Li MQ and Liu ZH: miRNA26a5p and miR26b5p inhibit the proliferation of bladder cancer cells by regulating PDCD10. Oncol Rep 40: 3523-3532, 2018.

32. Lu J, Zhang W, Ding Y, Li X and Song J: Expression of miR-26b in ovarian carcinoma tissues and its correlation with clinicopathology. Oncol Lett 17: 4417-4422, 2019.

33. Li D, Wei Y, Wang D, Gao H and Liu K: MicroRNA-26b suppresses the metastasis of non-small cell lung cancer by targeting MIEN1 via NF-kappaB/MMP-9/VEGF pathways. Biochem Biophys Res Commun 472: 465-470, 2016.

34. Liu J, Tu F, Yao W, Li X, Xie Z, Liu H, Li Q and Pan Z: Conserved miR-26b enhances ovarian granulosa cell apoptosis through HAS2-HA-CD44-Caspase-3 pathway by targeting HAS2. Sci Rep 6: 21197, 2016.

35. Kato M, Goto Y, Matsushita R, Kurozumi A, Fukumoto I, Nishikawa R, Sakamoto S, Enokida H, Nakagawa M, Ichikawa T and Seki N: MicroRNA-26a/b directly regulate La-related protein 1 and inhibit cancer cell invasion in prostate cancer. Int J Oncol 47: 710-718, 2015 .

36. Fukumoto I, Kikkawa N, Matsushita R, Kato M, Kurozumi A, Nishikawa R, Goto Y, Koshizuka K, Hanazawa T, Enokida H, et al: Tumor-suppressive microRNAs (miR-26a/b, miR-29a/b/c and miR-218) concertedly suppressed metastasis-promoting LOXL2 in head and neck squamous cell carcinoma. J Hum Genet 61 109-118, 2016

37. Liu SN, Li L, Li MH and Zhang JJ: Effect of miR-26b-5p on cis-diamine dichloroplatinum-induced ovarian granulosa cell injury by targeting MAP3K9. In Vitro Cell Dev Biol Anim 56: 213-221, 2020

(i) () () This work is licensed under a Creative Commons cc) $\mathrm{EY}$ No ND Attribution-NonCommercial-NoDerivatives 4.0 International (CC BY-NC-ND 4.0) License. 